\title{
Globally Optimal Channel Assignment for Non-cooperative Wireless Networks
}

\author{
Fan $\mathrm{Wu}$, Sheng Zhong, and Chunming Qiao \\ Department of Computer Science and Engineering \\ The State University of New York at Buffalo \\ Buffalo, NY 14260-2000, U.S.A. \\ Email: \{fwu2, szhong, qiao\}@ cse.buffalo.edu
}

\begin{abstract}
Channel assignment is a very important topic in wireless networks. In this paper, we study FDMA channel assignment in a non-cooperative wireless network, where devices are selfish. Existing work on this problem has considered Nash Equilibrium (NE), which is not a very strong solution concept and may not guarantee a good system-wide performance. In contrast, in this work we introduce a payment formula to ensure the existence of a Strongly Dominant Strategy Equilibrium (SDSE), a much stronger solution concept. We show that, when the system converges to a SDSE, it also achieves global optimality in terms of effective system-wide throughput. Furthermore, we extend our work to the case in which some radios have limited tunability. We show that, in this case, it is generally impossible to have a similar SDSE solution; but, with additional assumptions on the numbers of radios and the types of channels, etc., we can again achieve a SDSE solution that guarantees globally optimal effective system throughput in the entire system. Besides this extension, we also consider another extension of our strategic game, which is a repeated game that provides fairness. Finally, we evaluate our design in experiments. Our evaluations verify that the system does converge to the globally optimal channel assignment with our designed payment formula, and that the effective systemwide throughput is significantly higher than that of anarchy and Nash Equilibrium (NE).
\end{abstract}

\section{INTRODUCTION}

The radio spectrum is a scarce resource in this age of fast growing wireless communications. To better utilize the radio spectrum, Frequency Division Multiplexing Access (FDMA) is introduced to divide the carrier bandwidth into channels of different frequency, each carrying a signal at the same time. Some wireless systems also use Time Division Multiple Access (TDMA) or Carrier Sense Multiple Access with Collision Avoidance (CSMA/CA) to make it possible that multiple radio transceivers can access the same channel. With the emergence of software-defined radios, the problem of FDMA channel assignment has gained increasing importance. Here by channel assignment we mean the problem of assigning radio transceivers to available channels. Due to the limitation on the number of channels available, careful channel assignment is needed to mitigate the performance degradation of wireless networks because of interference.

In recent years, a large number of channel assignment schemes for wireless networks (e.g., [1]-[9]) have been proposed. In general, they assumed that all the nodes are "well behaved" or "cooperative". However, this assumption may not be valid in the current wireless networks [10]. In practice, a node can easily deviate from the protocol to seek for more

Fan Wu is supported by NSF CNS-0524030. Sheng Zhong is supported in part by NSF CNS-0524030. benefit for itself. So it is crucial to study how to provide incentives for the selfish nodes to behave cooperatively. In a recent work, Felegyhazi et al. [11] studied Nash Equilibria (NEs) in a non-cooperative multi-radio multi-channel allocation game. Here Nash Equilibrium (NE) is a standard solution concept from game theory. While their work is elegant and intriguing, $\mathrm{NE}$ as a solution concept does not provide an ideal solution in the problem of FDMA channel assignment. There are two reasons: (1) NE is not a very strong solution concept. When in a NE, a player of the game has incentives to keep its equilibrium strategy only under the assumption that all other players are also keeping their equilibrium strategies. When this assumption is not valid, NE does not provide incentives for the game player. (2) More important, NE is usually not social efficient, which means that the system-wide performance is not maximized. Therefore, when the system converges to one of the NEs, it could be the case that some of the selfish nodes benefit at the cost of system-wide performance degradation.

The objective of this paper is to use a much stronger solution concept - Strongly Dominant Strategy Equilibrium (SDSE), to guarantee that the system must converge to a state in which the effective system-wide throughput is optimized. By its definition (see Section III-B), SDSE ensures that, regardless of other nodes' behavior, a pair of communicating nodes always have incentives to use the strategy that maximizes the systemwide throughput. Hence the solution we provide is much stronger than any NE-based solution. The major contributions of this paper are as follows:

- First, we model the channel assignment problem as a strategic game. Our game model applies to the general scenario, where both single-radio devices and multi-radio devices can exist. By introducing a carefully designed payment formula (for using channels), we ensure the existence of a SDSE. Furthermore, we show that the SDSE achieves the global optimality in terms of effective system-wide throughput.

- Second, we extend our game model to a limited tunability system model and prove that one cannot find a similar SDSE. To deal with limited tunability, we introduce some practical assumptions, on the numbers of radios and on the types of channels, etc. With these assumptions, using another carefully designed payment formula, we can again have a SDSE that achieves the global optimality.

- Third, we study the fairness issue. We extend the strategic game to a repeated game. In our repeated game of channel assignment, not only the globally optimal effective 
throughput is preserved, but also the throughput shared among players are balanced in the long run.

- Finally, we evaluate our solutions using extensive experiments. Our experiments show that, with our designed payment formula, the system does converge to the globally optimal channel assignment. Compared with anarchy and $\mathrm{NE}$, the effective system-wide throughput is significantly higher.

The rest of the paper is organized as follows. We briefly review the related works in Section II and present the technical preliminaries in Section III. In Section IV, we describe our strategic game model of channel assignment, prove the existence of SDSE, and propose the algorithm for computing globally optimal channel assignment. We consider the limited tunability system model in Section V. In Section VI, we show a scheme to achieve fairness. And we present the evaluation results in Section VII. Finally, we conclude the paper and point out potential future works in Section VIII.

\section{RELATED WORKS}

In this section, we review the related works in this area. We first review the channel assignment works that assume cooperation of participants, and then review the works with selfish participants.

\section{A. Cooperated Channel Assignment Works}

Channel assignment problem was first studied in cellular networks. We refer to [1] for a comprehensive survey.

Due to explosive growth of wireless LANs (WLANs) in recent years, how to efficiently manage the channels becomes an important problem. For instance, Mishra et al. [2] utilize weighted graph coloring to address channel allocation for WLANs. Mishra et al. [3] use client-driven mechanisms to address the joint problem of channel assignment and load balancing in centrally managed WLANs.

As multi-radio devices are becoming more and more useful in wireless mesh networks (WMNs), many researchers devote themselves to studying channel assignment problems in WMNs. For example, Alicherry et al. [4], Raniwala et al. [5], and Kodialam et al. [6] consider channel assignment together with routing or scheduling in order to maximize network throughput. While the above works consider omnidirectional antennae, authors like in [7] consider the channel allocation problem in rural mesh networks that are built using directional antenna.

Channel assignment problem is also studied in other wireless networks, such as ad-hoc networks (e.g., [8]) and software defined radio networks (e.g., [9]).

\section{B. Channel Assignment Works with Selfish Participants}

The related works described in II-A require that all nodes in the network must be cooperative. However, this assumption is not valid when the network consists of selfish nodes. With the existence of selfish nodes, assigning radios to channels becomes a game.

In an existing work, Felegyhazi et al. [11] have studied Nash equilibria in a static multi-radio multi-channel allocation game. Their work is restricted to the scenario in which each device is equipped with the same number $(>1)$ of radios. In this paper, we seek for the existence of a much stronger solution concept called SDSE and give a scheme to achieve it. Further, our work is applicable to the general case, in which each wireless device can be equipped with an arbitrary number (possibly one) of radios.

Another important related work on channel assignment game is [12], in which the authors elegantly propose a graph coloring game model and discuss the price of anarchy under various topology conditions such as different channel numbers and bargaining strategies. Nevertheless, their work is restricted to the networks of base stations and requires the assumption that each base station has to choose a channel that has not been used by any other existing base stations. In contrast, our work does not have such assumptions.

In wireless networks, game theoretic approaches are also used to study media access problems. For example, MacKenzie et al. [13] study the selfish behavior of nodes in Aloha networks. Later, Cagalj et al. [14] and Konorski [15] use gametheoretic approach to investigate the media access problem of selfish nodes in CSMA/CA networks. In cognitive radio networks, Nie and Comaniciu [16] propose a game theoretic framework to analyze the behavior of cognitive radios for distributed adaptive spectrum allocation, but their main results are for cooperative users only.

There are also other works on incentive stimulation in wireless networks. Examples include those works on packet routing and forwarding in ad hoc networks [17]-[25].

\section{TEChNiCAl PRELIMINARIES}

\section{A. System Model}

We consider a wireless network, where nodes are equipped with one or more radios. Each radio has both a transmitter and a receiver which are combined in a single package, where the transmitter and the receiver may or may not be able to work simultaneously. We assume that the wireless network, whether it is infrastructure-based or infrastructure-less, has a common signal channel to facilitate the coordination among the nodes [8], [11]. We ignore the issue of coordination signal in the rest of this paper.

In the wireless network, there is a number of pairs of nodes who need to communicate with each other over single hop links. As in paper [11], we assume that each node participates in only one of the communication sessions at a time. To communicate, a pair of nodes allocate one or multiple radios. We assume that the transmission must be between two radios, where one acts as transmitter, and the other acts as receiver. So we only consider the case in which each node of the pair allocates the same number of radios in the same channel(s) . A pair of nodes can have parallel transmissions between them, if they both have multiple radios and allocate multiple radios.

The available frequency band is divided into orthogonal channels (e.g., IEEE 802.11a protocol [26] has 12 orthogonal channels). We denote the set of available orthogonal channels by $C$. We denote the effective aggregate throughput of a channel $c \in C$ by $R_{c}(n)$, where $n$ is the number of pairs of radio transmitter and receiver allocated to the channel c. $R_{c}(n)$ can be either a constant independent of $n$ or a decreasing function of $n$, corresponding to fixed-rate channels $C^{f}$ and varying-rate channels $C^{v}$, respectively. We note that $C=C^{f} \cup C^{v}$. For instance, $R_{c}(n)$ is independent of $n$ if TDMA based scheduling scheme is used; and $R_{c}(n)$ is a decreasing function when using CSMA/CA based protocol (e.g., IEEE 802.11 standards). As in paper [11], we assume 
that the effective aggregate throughput $R_{c}(n)$ of a channel $c$ is shared evenly among the radios using the channel. So each radio pair gets throughput $R_{c}(n) / n$, when $n>0$. (See [11] for why this assumption is valid in practice.)

In this paper, we only consider a single collision domain. This means that all users of a channel can hear each other's transmission on this channel. Extending our work to multiple collision domains requires consideration of the hidden terminal problem, which we leave for future study.

\section{B. Notations and Concepts from Game Theory}

Before introducing our game-theoretic model, we need to recall some notations from game theory. In the classic model of strategic game, there are a finite set of players $N=$ $\{1,2, \ldots, n\}$ and, for each player $i \in N$, a nonempty set $\Sigma_{i}$ of all possible (mixed) strategies. The set of strategy profiles is $\Sigma=\times_{i \in N} \Sigma_{i}$. Each player $i$ chooses a strategy $s_{i} \in \Sigma_{i}$. As a notational convention, $s_{-i}$ represents the strategies of all players except player $i$. Note that $s=\left(s_{i}, s_{-i}\right)$ is a strategy profile, in which player $i$ takes strategy $s_{i}$ and the other players take strategies $s_{-i}$. A player $i$ 's preferences can be determined by a utility function $u_{i}(s)$. Player $i$ prefers strategy $s_{i}$ to $s_{i}^{\prime}$ when the other players take $s_{-i}$, if $u_{i}\left(s_{i}, s_{-i}\right)>u_{i}\left(s_{i}^{\prime}, s_{-i}\right)$.

The most commonly used solution concept in game theory is Nash Equilibrium (NE) [27]:

Definition 1 (Nash Equilibrium): A Nash Equilibrium of a strategic game is a profile $s^{*} \in \Sigma$ of strategies with the property that for every player $i \in N$ we have

$$
u_{i}\left(s_{i}^{*}, s_{-i}^{*}\right) \geq u_{i}\left(s_{i}, s_{-i}^{*}\right),
$$

for all $s_{i} \in \Sigma_{i}$.

Although the Nash Equilibrium gives a fundamental solution concept to game theory, it relies on knowing all the other players' strategies and beliefs on the other players, and also loses power in the games where multiple NEs exist. A stronger solution concept is Strongly Dominant Strategy Equilibrium (SDSE) $)^{1}$ :

Definition 2 (Strongly Dominant Strategy Equilibrium): A Strongly Dominant Strategy Equilibrium of a strategic game is a profile $s^{*} \in \Sigma$ of strategies with the property that for every player $i \in N$,

$$
\begin{cases}\forall s_{-i} \in \Sigma_{i}, \forall s_{i} \neq s_{i}^{*}, & u_{i}\left(s_{i}^{*}, s_{-i}\right) \geq u_{i}\left(s_{i}, s_{-i}\right) \\ \exists s_{-i} \in \Sigma_{i}, \forall s_{i} \neq s_{i}^{*}, & u_{i}\left(s_{i}^{*}, s_{-i}\right)>u_{i}\left(s_{i}, s_{-i}\right) .\end{cases}
$$

\section{STRATEGIC GAME OF CHANNEL ASSIGNMENT}

We model the channel assignment problem as a strategic game $G$. We call it strategic game of channel assignment, in which a player is a pair of nodes having packets to exchange.

\section{A. Strategic Game Model}

In this paper, we consider a set $N$ of players, where each player $i$ knows her identity. In reality, a player's identity can be a quite long bit-string, like a MAC address. Nevertheless, for simplicity of presentation, in this paper we assume the players' identities are 1 through $n$. Note that our results are independent of this simplifying assumption. That is, all our results are still valid if the identities are not 1 through $n$. Each

\footnotetext{
${ }^{1}$ SDSE is related to, but different from, the well known concept of dominant strategy equilibrium (DSE). The major difference is that SDSE requires that, compared with any other strategy, the equilibrium strategy is strictly better in some cases.
}

player $i \in N$ has $w_{i}$ radio pairs. The radio pair distribution vector is denoted as $W=\left\{w_{1}, w_{2}, \ldots, w_{n}\right\}$.

In this game, the strategy of a player $i \in N$ is just her channel assignment vector $s_{i}=\left\{s_{i, 1}, s_{i, 2}, \ldots, s_{i, c}, \ldots, s_{i,|C|}\right\}$, where $s_{i, c}$ is the number of radio pairs that player $i$ assigns to channel $c$. The strategy profile $s$ is a matrix composed of all the players' strategies: $s=\left(s_{1}, s_{2}, \ldots, s_{n}\right)^{T}$.

Given a strategy profile $s$, it is easy to see the total number of radio pairs used by a player $i$ is $m_{i}(s)=\sum_{c \in C} s_{i, c} \leq$ $w_{i}$. Here, the inequality indicates that it is not necessary to use up all one's available radios. Similarly, it is also easy to see the total number of radios assigned to a channel $c$ is $n_{c}(s)=\sum_{i \in N} s_{i, c}$. Hence, the throughput a player $i$ gets from a channel $c$ is

$$
r_{i, c}(s)=\frac{s_{i, c}}{n_{c}} R_{c}\left(n_{c}\right),
$$

and the total throughput a player $i$ gets is

$$
r_{i}(s)=\sum_{c \in C} r_{i, c}
$$

Finally, the system-wide throughput is:

$$
T(s)=\sum_{c \in C} R_{c}\left(n_{c}\right) .
$$

In reality, any practical solution to the channel assignment game should satisfy some additional requirements. First of all, there should not be any starvation. Second, we need social efficiency, which means that the effective system-wide throughput should be maximized. We combine these two requirements to define the concept of global optimality of a solution ${ }^{2}$ :

Definition 3: (Global Optimality) In a strategic game of channel assignment, suppose that $s^{*}$ is a strategy profile or say a channel assignment. We say $s^{*}$ is globally optimal if the following two requirements are met:

1) No starvation. $\forall i \in N, r_{i}>0$.

2) Social efficiency. $\forall s \in A, s \neq s^{*}$, if $s$ satisfies requirement (1), then $T(s) \leq T\left(s^{*}\right)$.

We note that the globally optimal channel assignment might not be unique. But all globally optimal channel assignments have the same overall throughput in the system.

\section{B. Method to Achieve Global Optimality}

It is ideal to have a globally optimal channel assignment. However, achieving the globally optimal channel assignment is a highly challenging task. If we allow the players to choose the channels without giving them any influence, most likely the system would either not converge at all, or converge to an assignment that is not globally optimal. Therefore, we need to introduce a method to influence the strategies of the players. Here the method we use is to require players to make payments.

Just as in [19]-[25], we assume that there is some kind of virtual currency in the system. Each player has to pay some virtual money to the system administrator based on the outcome of the strategy profile. We regard this payment as the

\footnotetext{
${ }^{2}$ Our definition of global optimality is thus slightly different from a traditional definition, which usually considers the optimization of a single metric (e.g., throughput).
} 
fee for using the channels. Note that the system administrator need not to be an online authority. It is just a server connected to the Internet. So the players can pay or receive credit from the system administrator when they have connections to the Internet.

Now let's assume we have a globally optimal strategy profile $s^{*}$ (We will explain how to compute $s^{*}$ in Section IV-C.). We define the payment of player $i$ as follows:

$$
p_{i}=\alpha r_{i}+\beta\left(D\left(s_{i}, s_{i}^{*}\right)-\frac{1}{n-1} \sum_{j \in N, j \neq i} D\left(s_{j}, s_{j}^{*}\right)\right),
$$

where $D\left(s_{i}, s_{i}^{*}\right)$ is the Manhattan distance (also known as the L1-distance) between strategies $s_{i}$ and $s_{i}^{*} ; \alpha>0$ and $\beta>0$ are parameters used for converting throughput and the Manhattan distance into virtual currency values, respectively. Intuitively, the payment is the charge for the player's overall throughput plus a penalty (bonus) for more (less) deviation from the globally optimal strategy than other players. We note that the total payments to the system administrator is:

$$
P=\sum_{i \in N} p_{i}=\alpha \sum_{i \in N} r_{i}
$$

which is the value of total throughput shared by the players. We further note that if all the channels are used,

$$
P=\alpha \sum_{i \in N} r_{i}=\alpha \sum_{c \in C} R_{c}\left(n_{c}\right),
$$

which is the value of effective system-wide throughput.

We define the utility of player $i$ as the value of throughput she obtains minus her payment to the SA:

$$
u_{i}=\alpha r_{i}-p_{i}
$$

Since each player is selfish and rational, she always wants to maximize her utility.

Theorem 4: It is a SDSE when each player $i$ takes strategy $s_{i}^{*}$. Furthermore, if $s^{*}$ is a globally optimal channel assignment, then the SDSE achieved is also globally optimal.

Proof: Combining Equation (6) and (7), we can get:

$$
u_{i}=-\beta\left(D\left(s_{i}, s_{i}^{*}\right)-\frac{1}{n-1} \sum_{j \in N, j \neq i} D\left(s_{j}, s_{j}^{*}\right)\right) .
$$

Then the utility difference of taking strategy $s_{i}^{*}$ and $s_{i} \neq s_{i}^{*}$ is:

$$
\begin{aligned}
u_{i}^{*}-u_{i}= & -\beta\left(D\left(s_{i}^{*}, s_{i}^{*}\right)-\frac{1}{n-1} \sum_{j \in N, j \neq i} D\left(s_{j}, s_{j}^{*}\right)\right) \\
& +\beta\left(D\left(s_{i}, s_{i}^{*}\right)-\frac{1}{n-1} \sum_{j \in N, j \neq i} D\left(s_{j}, s_{j}^{*}\right)\right) \\
= & \beta\left(D\left(s_{i}, s_{i}^{*}\right)-D\left(s_{i}^{*}, s_{i}^{*}\right)\right) \\
= & \beta D\left(s_{i}, s_{i}^{*}\right) \\
> & 0 .
\end{aligned}
$$

So strategy profile $s^{*}$ is a SDSE.

\section{Algorithm for Computing Globally Optimal Channel As- signment}

To implement the SDSE, each player must have an algorithm for computing the globally optimal assignment $s^{*}$. In this subsection, we give an algorithm that computes a globally optimal channel assignment $s^{*}$, if there exists one. The input of this algorithm is the set of channels $C$, the set of players $N$, and the radio distribution vector $W$.

Algorithm 1 Algorithm for Computing Globally Optimal
Channel Assignment

Input: Set of channels $C=C^{f} \cup C^{v}$, set of players $N$, radio distribution vector $W$.

Output: Globally optimal channel assignment $s^{*}$.

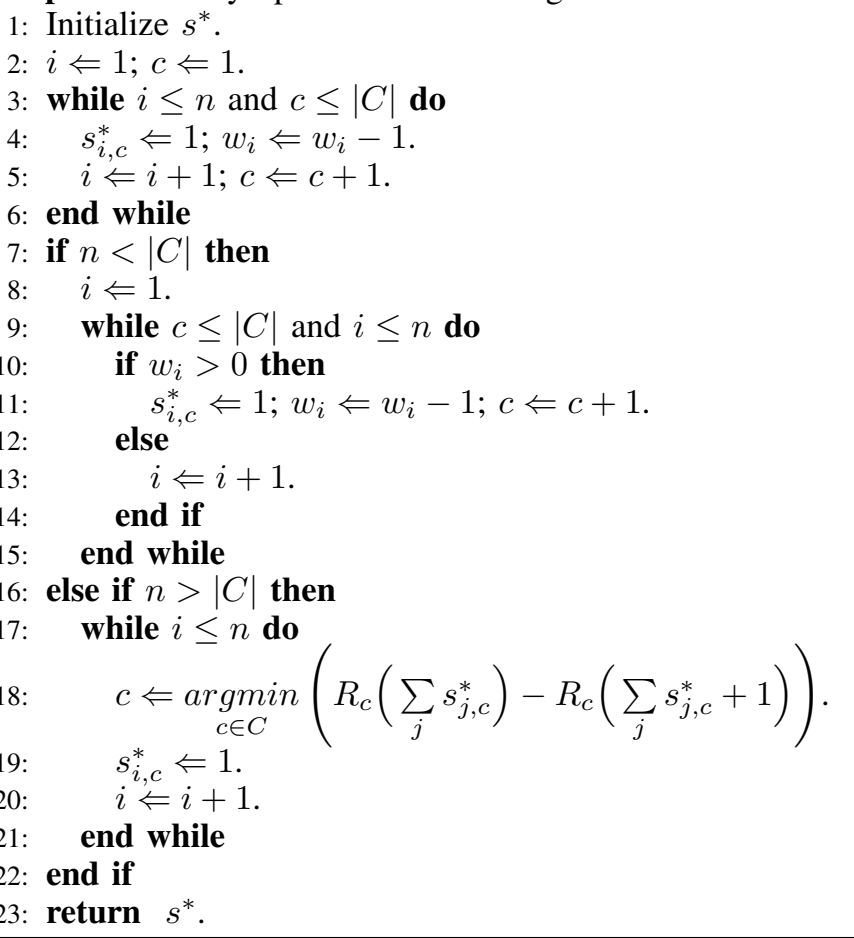

Algorithm 1 shows the pseudo-code of our algorithm. Intuitively, the algorithm considers three cases: (1) The number of players is less than that of channels. (2) The number of players is more than that of channels. (3) The number of players and that of channels are equal. For all the cases, the algorithm first assigns each player with a single channel. Next, in case (1), the algorithm tries to assign each unoccupied channel with a player who still has unused radio pair, until all the channels are occupied or all the radios of players are used. In case (2), for each unassigned player $i$, the algorithm finds a channel $c$ on which adding a radio pair will cause the least throughput degradation. Then it assigns player $i$ with channel $c$. In case (3), we are done with channel assignment and the algorithm terminates.

\section{LIMITED TUNABILITY}

In previous sections, we have considered the case in which all radios have unlimited tunability and thus have full access to all channels. In reality, since the wireless networks usually consist of various devices (e.g., laptop/desktop PC, PDA, IP phone), the radios of the devices may not have the tunability 
to access all the channels. In this section, we extend our work to the case in which some players have limited tunability. Here we say a player can be tuned to, or can access, a channel if both nodes of the player can send/receive signals in that channel. (Recall that the two nodes of each player must assign the same number of radios to each channel.) Note that the problem in limited tunability model is much more challenging than the one in unlimited tunability model. For example, selfish players may not willing to reveal their real tunability information, which complicates the problem a lot. So, it is not surprising that our first result in the limited tunability model is a result of non-existence of SDSE solution.

\section{A. Non-existence of SDSE Solution}

With limited tunability of radios, the first result we obtain is that we can no longer have a SDSE solution as in the case of unlimited tunability.

Theorem 5: Even if there is only a single player who can not access all the channels (and can claim arbitrarily which channels it can access), there is always a scenario in which no SDSE satisfies the requirements below:

1) Each player claims her tunability honestly.

2) The assignment of channels in the equilibrium is globally optimal.

Proof: We prove by contradiction. Assume this is not true. Then, we consider a game of two players (1 and 2) and two channels $\left(c_{1}\right.$ and $\left.c_{2}\right)$. We study two scenarios $S_{1}$ and $S_{2}$ as follows.

In scenario $S_{1}$, both players can access both channels. As we have assumed, there must be a SDSE $s^{*}$ satisfying the above two requirements. Clearly, $s^{*}$ must assign each of the two channels to each of the two players. Without loss of generality, suppose that $s^{*}$ assigns channel $c_{1}$ to player 1 and channel $c_{2}$ to player 2 .

Now we construct a different scenario $S_{2}$ based on the above assignment of $s^{*}$. In $S_{2}$, player 2 can access channel $c_{1}$ only, while player 1 can still access both channels. By our assumption, in this scenario we also have a SDSE $s^{\prime}$ satisfying the two requirements. Note that $s^{\prime}$ must assign $c_{1}$ to player 2 since otherwise player 2 would starve. Then, it is easy to see that $s^{\prime}$ assigns $c_{2}$ to player 1 . Clearly, we can see $s^{\prime} \neq s^{*}$.

Next, recall that the utility of a player only depends on the strategy profile. So, denote by $u_{2}(s)$ the utility of player 2 when the strategy profile is $s$. Since $s^{*}$ is a SDSE in scenario $S_{1}$, there exists $s_{1}$ such that

$$
u_{2}\left(s_{1}, s_{2}^{*}\right)>u_{2}\left(s_{1}, s_{2}^{\prime}\right) .
$$

On the other hand, since $s^{\prime}$ is a SDSE in scenario $S_{2}$, we always have

$$
u_{2}\left(s_{1}, s_{2}^{*}\right) \leq u_{2}\left(s_{1}, s_{2}^{\prime}\right) .
$$

We get a contradiction from Equations (9) and (10).

\section{B. Simplified Model and Solution}

Given Theorem 5, to achieve a SDSE solution, we have to simplify our model to make the problem more tractable. Consequently, we assume that each player has only one pair of radios; all the channels are fixed-rate channels; and the majority of players can access all channels. If a number of players detect that a player is cheating about her tunability,
1) Each player $i \in N$ sends test signals in each channel it claims to be able to access. We denote the accessible channel set of player $i$ as $T_{i}$.

2) After receiving all the test signals, the players with full accessibility compute the globally optimal channel assignment $s^{*}$ and broadcast it in all channels.

3) Each player $i$ takes strategy $s_{i}$ and pays two payments:

$$
\begin{aligned}
p_{i}^{1}= & \alpha r_{i}+\beta\left(D\left(s_{i}, s_{i}^{*}\right)\right. \\
& \left.-\frac{1}{n-1} \sum_{j \in N, j \neq i} D\left(s_{j}, s_{j}^{*}\right)\right), \\
p_{i}^{2}= & \gamma\left(|C|-\left|T_{i}\right|\right),
\end{aligned}
$$

here $\gamma$ is a charge for inaccessibility to a channel and $\gamma>4 \beta$.

Fig. 1. Scheme for achieving SDSE in the simplified limited tunability model.

then the latter player will be punished by an overwhelming penalty.

In this simplified model, again, we assume that we have an algorithm for computing the globally optimal channel assignment. (Note that such an algorithm is different from the one in Section IV-C, since the model is now different. We will discuss this new algorithm in Section V-C.) We design a scheme (see Figure 1) that ensures the existance of a SDSE that achieves global optimality. In our scheme, to claim the accessibility to a channel, a player needs to send a test signal in that channel ${ }^{3}$, so that other players can verify her claim. In this way, a player has no way to exaggerate her accessible channels. So a player can only claim a subset of her real accessible channels. Recall that the majority of the players have unlimited tunability. Consequently, the above test signal can be verified by most players. The following lemma shows that by claiming a proper subset of one's accessible channels, a player will lose her utility. In other words, a player maximize her expected utility only by revealing the true tunability.

Lemma 6: Other things being equal, if our scheme is used, for every player, revealing the true tunability is always better than claiming a proper subset of accessible channels.

Proof: Suppose a player $i$ claims her accessible channel set $T_{i}^{\prime} \subset T_{i}$ and gets utility $u_{i}^{\prime}$. We show that $u_{i}^{\prime}$ is always less than $u_{i}$, which is the utility when claiming the true accessible channel set $T_{i}$.

$$
\begin{aligned}
u_{i}-u_{i}^{\prime}= & \alpha r_{i}-p_{i}^{1}-p_{i}^{2}-\left(\alpha r_{i}^{\prime}-p_{i}^{\prime 1}-p_{i}^{\prime 2}\right) \\
= & -\beta\left(D\left(s_{i}, s_{i}^{*}\right)-\frac{1}{n-1} \sum_{j \neq i} D\left(s_{j}, s_{j}^{*}\right)\right) \\
& +\beta\left(D\left(s_{i}^{\prime}, s_{i}^{\prime *}\right)-\frac{1}{n-1} \sum_{j \neq i} D\left(s_{j}^{\prime}, s_{j}^{\prime *}\right)\right) \\
& -\gamma\left(|C|-\left|T_{i}\right|\right)+\gamma\left(|C|-\left|T_{i}^{\prime}\right|\right)
\end{aligned}
$$

${ }^{3}$ Here the test signal we mentioned is actually a pair of signals sent by the pair of radios of the player. 
Since each player only has one radio pair,

$$
0 \leq D\left(s_{x}, s_{y}\right) \leq 2
$$

for all $s_{x}, s_{y}$.

Combine Equation (13) and (14), we get

$$
\begin{aligned}
u_{i}-u_{i}^{\prime} & \geq \gamma\left(\left|T_{i}\right|-\left|T_{i}^{\prime}\right|\right)-4 \beta \\
& \geq \gamma-4 \beta
\end{aligned}
$$

Since $\gamma>4 \beta$,

$$
u_{i}-u_{i}^{\prime}>0
$$

Now it is not hard to show that we have a SDSE in which each player claims her true tunability and uses the (computed) strategy for globally optimal assignment.

Theorem 7: There exists a SDSE in the simplified model of limited tunability such that each player claims the true tunability and that the channel assignment is globally optimal.

\section{Algorithm for Computing the Globally Optimal Channel} Assignment in the Simplified Model of Limited Tunability

To implement the scheme in Section V-B, we need an algorithm for computing the globally optimal channel assignment in our simplified model of limited tunability. Just as Algorithm 1, this algorithm also has the player set $N$ and the channel set $C$ as the input. It does not need the radio distribution vector because the radio distribution vector is a constant in our simplified model. In addition, the algorithm takes as input of an accessability vector $\left(X=\left(X_{1}, X_{2}, \ldots, X_{i}, \ldots, X_{n}\right)\right.$, where $X_{i} \subseteq C$ ) that indicates which player can access which channel(s). Based on this information, the algorithm needs to compute a globally optimal channel assignment.

We convert the problem to a graph theoretic problem. Construct a vertex set $V_{1}$ by having a vertex for each player. Construct another vertex set $V_{2}$ by having a vertex for each channel. If a player can access a channel, then these two vertices are connected together by an edge - let $E$ be the set of such edges. In this way, we get a bipartite graph $G=\left(V_{1} \cup V_{2}, E\right)$. A channel assignment corresponds to a subset of edges such that each player is associated with only one channel through this subset. We note that we can map each channel assignment to a matching in the graph: in the subset of edges corresponding to the assignment, for each channel assigned to more than one players, we keep one edge and delete the others; in this way, we get a subset of edges that is a matching, and this matching's aggregate throughput is the same as the original assignment. (However, we note that more than one assignments may map to the same matching.) Therefore, a globally optimal channel assignment is mapped to a maximum bipartite matching in the bipartite graph. Assume $\operatorname{MBM}\left(V_{1}, V_{2}, E\right)$ is a deterministic algorithm for maximum bipartite matching ${ }^{4}$. We let all players must use the same $M B M$ algorithm to find a maximum matching. When there is more than one maximum matching, the $M B M$ algorithm should choose to output one of them. Clearly, all players will get the same maximum matching because they are using the same algorithm and the algorithm is deterministic.

Algorithm 2 shows the pseudo-code of our algorithm in the simplified limited tunability model. First, the algorithm

\footnotetext{
${ }^{4}$ See [28] for examples of such algorithms.
}

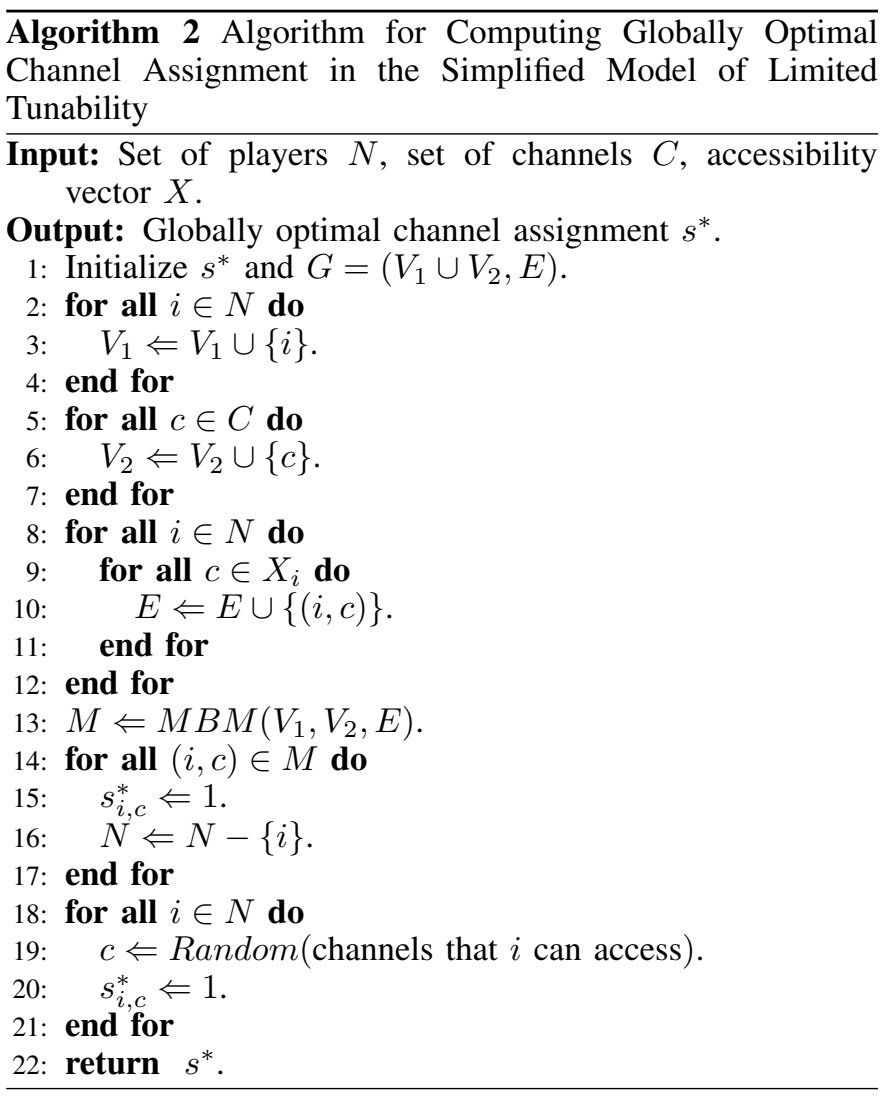

transforms the channel assignment problem on $N$ and $C$ to a maximum bipartite matching problem on graph $G=$ $\left(V_{1} \cup V_{2}, E\right)$. Then the algorithm calls a existing algorithm for finding the maximum matching. Next, it computes the channel assignment based on the maximum matching. Finally, to ensure there is no starvation, for each player that has not been assigned a channel in the matching, the algorithm assign an arbitrary channel to her.

\section{FAIRness Through REPEATED GAME}

In the previous sections, we have studied globally optimal channel assignment in strategic games, which guarantees no starvation and the highest effective system throughput. However, in some applications, fairness may be more crucial than effective system throughput. Therefore, we extend our work to a different game theoretic model, so that we can obtain the maximum amount of fairness.

In this section, we consider channel assignment as a repeated game ${ }^{5}$. Essentially, this repeated game models a situation in which players repeatedly engage in the strategic game $G$ as defined in Section IV. There is no limitation on the number of times that $G$ is played; and in each round the players take their strategies simultaneously. Using the terminology of repeated game, each round of strategic game here is called a stage. All the stages have the same length of

\footnotetext{
${ }^{5}$ Note that our model of repeated game is slightly different from the standard model of repeated game from game theory. In the standard model, the utility function is fixed in each stage of the game. However, in our model, the definition of payment is based on the globally optimal channel assignment Consequently, the payment formula changes along with the globally optimal channel assignment in each stage of the game. So does the utility function.
} 
time. We treat each stage as a strategic game. Furthermore, we define the cumulative utility of player $i \in N$ from the beginning of the game to stage $t$ as:

$$
\hat{u}_{i}(t)=\sum_{j=0}^{t} u_{i}(j) .
$$

Let us assume, as in Section IV, that all radios have unlimited tunability. We further assume that each player has the same number of radios in the repeated game. Then, we can get a completely fair channel assignment as follows.

In stage $t$, we define a channel assignment matrix $s^{*}(t)$, which will be used to compute the payments to the system administrator.

$$
s^{*}(t)= \begin{cases}s^{*} & \text { if } t=0 \\
\left(\begin{array}{c}
s_{2}^{*}(t-1) \\
\ldots \\
s_{n}^{*}(t-1) \\
s_{1}^{*}(t-1)
\end{array}\right) & \text { if } t>0,\end{cases}
$$

where $s^{*}$ is a SDSE we defined in Section IV.

Since each player's utility and the system-wide throughput in a stage are always independent from other stages, we can easily obtain the following theorem:

Theorem 8: Each player $i$ gets its cumulative utility maximized when taking the strongly dominant strategy $s_{i}^{*}(t)$ in each stage $t$.

Finally, we show that we can achieve complete fairness in the repeated game.

Theorem 9: In our repeated game of channel assignment, if every player takes the dominant strategy in each stage, then each player gets the same throughput in the long run.

Proof: In the repeated game, suppose player $i$ takes the dominant strategy $s_{i}^{*}(t)$ in each stage. The average throughput that player $i$ gets from the beginning of the game to stage $t$ is:

$$
\begin{aligned}
\bar{r}_{i}(t) & =\frac{1}{t} \sum_{j=0}^{t} r_{i}(j) \\
& =\frac{1}{t} \sum_{j=0}^{t} r_{(i+j) \bmod n}(0) .
\end{aligned}
$$

Consider the infinity of the repeated game:

$$
\begin{aligned}
\lim _{t \rightarrow+\infty} \bar{r}_{i}(t) & =\lim _{t \rightarrow+\infty} \frac{1}{t} \sum_{j=0}^{t} r_{(i+j) \bmod n}(0) \\
& =\lim _{t \rightarrow+\infty} \frac{1}{t} \cdot \frac{t}{n} \sum_{j=0}^{n-1} r_{i+j}(0) \\
& =\frac{1}{n} \sum_{j=1}^{n} r_{j}(0) .
\end{aligned}
$$

This completes the proof.

\section{Evaluations}

In this section, we evaluate our schemes using MATLAB. We assume that the available frequency band is divided into 12 orthogonal channels, which consist of fixed-rate channels and varying-rate channels. In the evaluations, basic CSMA/CA protocol with binary slotted exponential backoff is used for varying-rate channels. We use the same system parameters as in paper [29]. We assume that the channel bit rate is $1 \mathrm{Mbit} / \mathrm{s}$. Besides, we set $\alpha=\beta=1$ and $\gamma=5$.

\section{A. Evaluations in the Unlimited Tunability Model}

We have two sets of simulations done in the unlimited tunability model. The first one is to compare the effective system throughput of SDSE achieved by using our scheme with anarchy and NE. Here anarchy and NE do not charge the players for using the channels. The difference between anarchy and NE is: anarchy is a state where players arbitrarily assign their radio(s) to the channels; while NE is a relatively stable state, in which no player can increase her throughput by reassigning her radio(s). The second one is to show that if our scheme is used, deviating from the computed channel assignment can not increase one's utility.

In the first set of simulations, we consider three different deployments of channels: (1) no varying-rate channel, (2) 8 fixed-rate channels and 4 varying-rate channels, and (3) no fixed-rate channel. We vary the number of players from 2 to 40 with interval 2 . The number of radio pairs each player has is uniformly distributed in $[1,5]$. We repeat the simulation 100 times, and average the results.

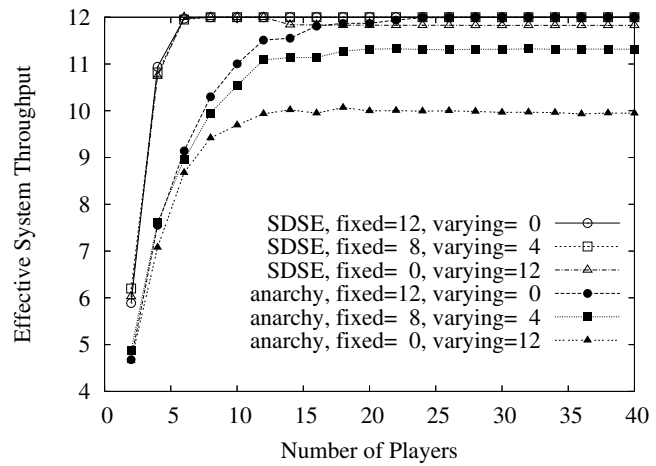

Fig. 2. Effective system throughput of the SDSE achieved by using our scheme and anarchy.

Figure 2 shows the result of the comparison between the SDSE achieved by using our scheme and anarchy. Generally, the SDSE reaches the maximum effective system throughput as long as there are only a few players. As Figure 2 shows, in all the three cases, the SDSE reaches effective system throughput $12 \mathrm{Mbit} / \mathrm{s}$ with only 8 players. But when there exist varying-rate channels, the effective system throughput of anarchy will never reach $12 \mathrm{Mbit} / \mathrm{s}$. Even without varyingrate channel, the anarchy can get $12 \mathrm{Mbit} / \mathrm{s}$ only when there are at least 28 players in the system. Another advantage of the SDSE is that it causes much less system degradation than the case of anarchy, when there exist varying-rate channels. In case (2), the SDSE achieves $0.68 \mathrm{Mbit} / \mathrm{s}$ more effective system throughput than anarchy in most cases, while in case (3), the difference of effective system throughput is as high as $1.76 \mathrm{Mbit} / \mathrm{s}$ or even more.

Figure 3 demonstrates the comparison result between the SDSE achieved by using our scheme and a NE. Since there is no system degradation when no varying-rate channel exists, we only show the later two cases here. When the resource 


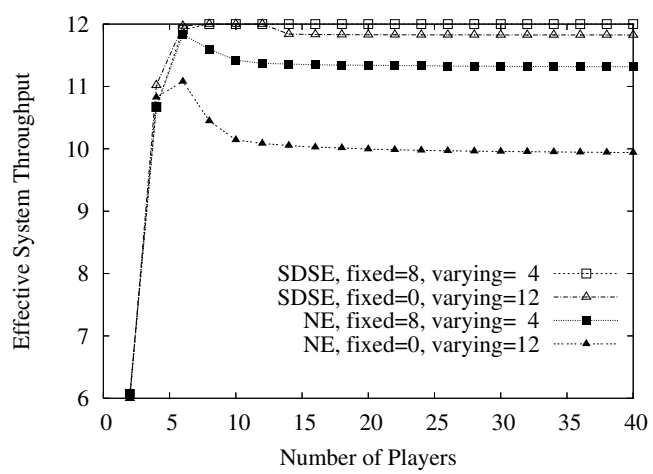

Fig. 3. Effective system throughput of SDSE achieved by using our scheme and NE.

(channels) is abundant (less or equal to 4 players with average of 3 radio pairs), the NE gets almost the same effective system throughput as the SDSE. But when the resource is scarce, the greedy nature of the players will make the contention for the channels hotter and hotter with the growth of players, regardless of system degradation. So the SDSE performs much better than the NE, when the resource is scarce. When there are 40 players, the effective system throughput of our SDSE is $0.68 \mathrm{Mbit} / \mathrm{s}$ higher than that of the NE for case (2), and $1.89 \mathrm{Mbit} / \mathrm{s}$ higher for case (3).

Our second set of simulations demonstrates the effect of deviating from our scheme. In this set of simulations, we assume there are 40 players in the system, and $50 \%$ of them deviate from our scheme by arbitrarily assigning their radios to the channels. The other setups are the same as the first set of simulations. The simulation is also repeated 100 times. We keep track of a player and record her utility in the two cases: following our scheme or deviating from it.

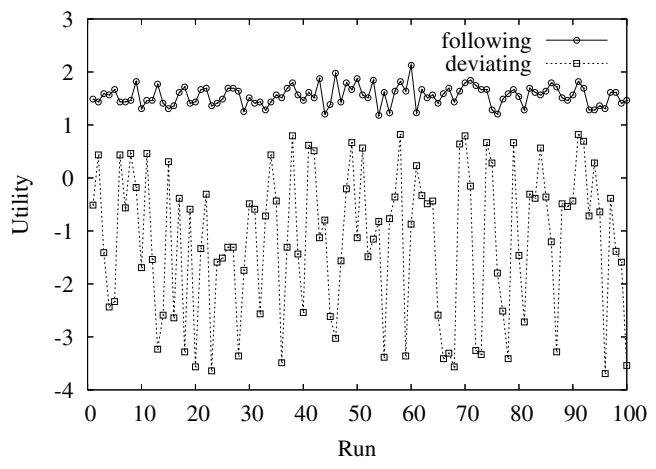

Fig. 4. Utility of following our scheme and deviating.

Figure 4 illustrates the utility of the tracked player. It is shown that, when following our scheme, the player can always gets non-negative utility in any run. Furthermore, the utility curve of following the scheme is always above that of deviating. So following our scheme is clearly better than deviating from it.

\section{B. Evaluations in the Limited Tunability Model}

We also do two sets of simulations in the limited tunability model. The first one compares the effective system throughput of SDSE achieved by using our scheme with anarchy, while the second one studies the effect of cheating about one's tunability and deviating from the computed channel assignment.

In the first set of simulations, we consider two different ratios of players who have limited tunability: $20 \%$ and $40 \%$. For the players with limited tunability, we restrict her number of accessible channels uniformly between 1 and 11 . We vary the number of players from 2 to 40 with interval 2, and repeat each simulation 100 times.

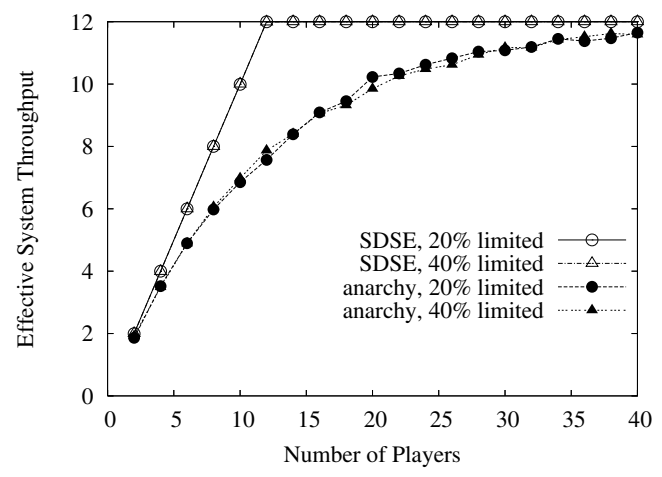

Fig. 5. Effective system throughput of the SDSE achieved by using our scheme and anarchy in the limited tunability model.

Figure 5 illustrates the comparison on effective system throughput between the SDSE achieved by using our scheme and anarchy. The effective system throughput of our SDSE grows almost linearly when no more than 12 players in the network, and remains at maximum after that. Compared with anarchy, which never reaches $12 \mathrm{Mbit} / \mathrm{s}$, our SDSE obviously has better effective system throughput.

In the second set of simulations, we observe the effect of cheating about tunability and deviating from the computed channel assignment. We calculate the utility of following our scheme minus the utility of cheating and deviating

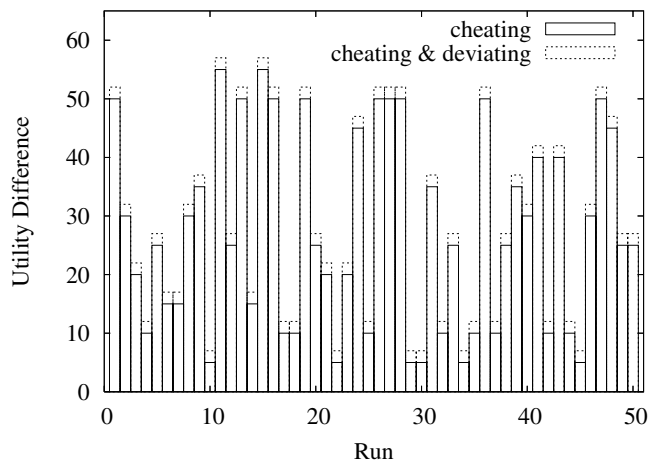

Fig. 6. Utility difference between following our scheme and cheating \& deviating in the limited tunability model.

In Figure 6, we can see that the utility difference is always higher than zero in 50 iterations. And we observe that compared with deviating, cheating is clearly the dominant source of utility loss. So it is always better for the players to claim their true tunability and follow the computed channel assignment.

\section{Evaluations on the Repeated Game}

In this set of evaluations, we assume there are 8 fixed-rate channels and 4 varying-rate channels. The number of players 
is set to 40 . Each player has 2 radio pairs. We let the repeated game go 4000 stages and record the standard deviation of players' average throughput in each stage.

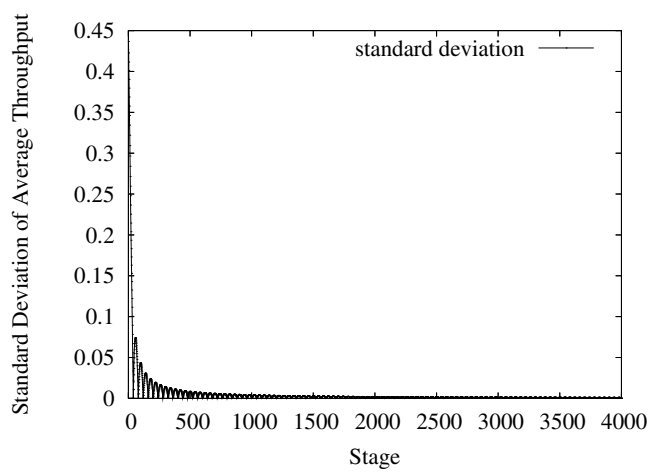

Fig. 7. Standard deviation of players' average throughput with growth of the stage.

From Figure 7, we observe that there is a cycle of 40 stages. At the end of each cycle, the standard deviation gets zero. Each cycle has a peak, which goes down towards zero with progress of the repeated game.

\section{CONCLUSION AND FUTURE WORK}

In this paper, we study the channel assignment problem in non-cooperative wireless networks. We model the channel assignment problem as a strategic game and guarantee the existence of a SDSE by introducing a payment formula. Furthermore, the SDSE achieves the global optimality in terms of effective system-wide throughput. Next, we prove that the above result does not hold in the general limited tunability model. But, when we make additional practical assumptions on the numbers of radios and the types of channels, etc., we can achieve a SDSE solution that guarantees globally optimal effective system throughput again. To get better fairness, we extend the strategic game to a repeated game, which preserve the global optimality in each stage and achieves fairness in the long run.

There are several potential ways to extend our work. One possibility is to study the tradeoff among effective system-wide throughput, fairness among the players, and load-balance on the channels. Another possibility is to consider strategic game of channel assignment in multiple collision domain. However, we leave these topics to future study.

\section{REFERENCES}

[1] I. Katzela and M. Naghshineh, "Channel assignment schemes for cellular mobile telecommunications: A comprehensive survey," IEEE Personal Communications, vol. 3(3), pp. 10-31, Jun. 1996.

[2] A. Mishra, S. Banerjee, and W. Arbaugh, "Weighted coloring based channel assignment for WLANs," ACM SIGMOBILE Mobile Computing and Communications Review (MC2R), vol. 9, no. 3, pp. 19-31, 2005.

[3] A. Mishra, V. Brik, S. Banerjee, A. Srinivasan, and W. Arbaugh, "A client-driven approach for channel management in wireless LAN," in IEEE INFOCOM '06, Apr. 2006.

[4] M. Alicherry, R. Bhatia, and L. Li, "Joint channel assignment and routing for throughput optimization in multi-radio wireless mesh networks," in ACM MobiCom '05, Aug. 2005.

[5] A. Raniwala, K. Gopalan, and T. cker Chiueh, "Centralized channel assignment and routing algorithms for multi-channel wireless mesh networks," ACM SIGMOBILE Mobile Computing and Communications Review (MC2R), vol. 8, no. 2, pp. 50-65, 2004.
[6] M. Kodialam and T. Nandagopal, "Characterizing the capacity region in multi-radio multi-channel wireless mesh networks," in ACM MobiCom '05, Aug. 2005.

[7] B. Raman, "Channel allocation in 802.11-based mesh networks," in IEEE INFOCOM '06, Apr. 2006.

[8] P. Kyasanur and N. Vidya, "A routing protocol for utilizing multiple channels in multi-hop wireless networks with a single transceiver," in QSHINE '05, Aug. 2005.

[9] Y. T. Hou, Y. Shi, and H. D. Sherali, "Optimal spectrum sharing for multi-hop software defined radio networks," in IEEE INFOCOM '07, May 2007.

[10] M. Raya, J.-P. Hubaux, and I. Aad, "DOMINO: a system to detect greedy behavior in ieee 802.11 hotspots," in MobiSys '04, 2004.

[11] M. Félegyházi, M. Čagalj, S. S. Bidokhti, and J.-P. Hubaux, "Noncooperative multi-radio channel allocation in wireless networks," in IEEE INFOCOM '07, May 2007.

[12] M. M. Halldórsson, J. Y. Halpern, L. E. Li, and V. S. Mirrokni, "On spectrum sharing games," in ACM PODC '04, Jul. 2004.

[13] A. B. MacKenzie and S. B. Wicker, "Stability of multipacket slotted Aloha with selfish users and perfect information," in IEEE INFOCOM '03, Mar. 2003.

[14] M. Cagalj, S. Ganeriwal, I. Aad, and J.-P. Hubaux, "On selfish behavior in CSMA/CA networks," in IEEE INFOCOM '05, Mar. 2005.

[15] J. Konorski, "Multiple access in ad-hoc wireless lans with noncooperative stations," in Networking '02, May 2002.

[16] N. Nie and C. Comaniciu, "Adaptive channel allocation spectrum etiquette for cognitive radio networks," in DySPAN '05, Nov. 2005

[17] L. Anderegg and S. Eidenbenz, "Ad hoc-vcg: a truthful and cost-eftcient routing protocol for mobile ad hoc networks with seltsh agents," in $A C M$ MobiCom '03, Jun. 2003.

[18] V. Srinivasan, P. Nuggehalli, C.-F. Chiasserini, and R. Rao, "Cooperation in wireless ad hoc wireless networks," in IEEE INFOCOM '03, Mar. 2003.

[19] W. Wang, X. Y. Li, and Y. Wang, "Truthful multicast in selfish wireless networks," in ACM MobiCom '04, Sep. 2004.

[20] S. Zhong, L. Li, Y. G. Liu, and Y. R. Yang, "On designing incentivecompatible routing and forwarding protocols in wireless ad-hoc networks,' in ACM MobiCom '05, Aug. 2005.

[21] S. Zhong, J. Chen, and Y. R. Yang, "Sprite: a simple, cheat-proof, creditbased system for mobile ad-hoc networks," in IEEE INFOCOM '03, Mar. 2003.

[22] W. Wang, S. Eidenbenz, Y. Wang, and X. Y. Li, "Ours: Optimal unicast routing systems in non-cooperative wireless networks," in $A C M$ MobiCom '06, Sep. 2006.

[23] N. Salem, L. Buttyan, J. Hubaux, and M. Jakobsson, "A charging and rewarding scheme for packet forwarding in multi-hop cellular networks," in ACM MobiHoc '03, Jun. 2003.

[24] S. Eidenbenz, G. Resta, and P. Santi, "Commit: A sender-centric truthful and energy-efficient routing protocol for ad hoc networks with selfish nodes," in IEEE IPDPS '05, Apr. 2005.

[25] S. Zhong and F. Wu, "On designing collusion-resistant routing schemes for non-cooperative wireless ad hoc networks," in ACM MobiCom '07, Sep. 2007.

[26] IEEE 802.11a Working Group, "Wireless LAN medium access control (MAC) and physical layer (PHY) specifications - amendment 1: Highspeed physical layer in the $5 \mathrm{GHz}$ band," 1999.

[27] M. J. Osborne and A. Rubenstein, A Course in Game Theory. The MIT Press, 1994

[28] T. H. Cormen, C. E. Leiserson, R. L. Rivest, and C. Stein, Introduction to Algorithms, Second Edition. Section 26.3: Maximum bipartite matching. MIT Press and McGraw-Hill, 2001.

[29] G. Bianchi, "Performance analysis of the IEEE 802.11 distributed coordination function," IEEE Journal on Selected Areas in Communications, vol. 18 , no. 3, pp. 535-547, 2000. 